\title{
Influences of Anti-G Suit Material Hygroscopicity on Thermal Stress Index of the Pilot
}

\author{
LI Fengzhi ${ }^{1, a}$, REN Penghao ${ }^{2, b}$ \\ ${ }^{1,2}$ College of Aerospace Engineering, Nanjing University of Aeronautics and Astronautics, Nanjing, \\ 210016, China \\ ahelifz@nuaa.edu.cn, b863741949@qq.com
}

Keywords: Anti-G Suit; Thermal Stress Index; Heat and Moisture Transfer; Model; Simulation

\begin{abstract}
To investigate the influences of the fiber hygroscopicity on the pilot thermal responses, a heat and moisture transfer model for pilot/anti-G suit/environment system is developed. An improved Stolwijk model is used to simulate pilot thermal responses, and the coupled heat and moisture transfer model considering fiber hygroscopicity role is used for anti-G suit. The model is validated by literature experimental data. Then, effects of the fiber hygroscopicity on the pilot thermal stress index are analyzed by running the model program. Conclusion shows that the anti-G suit made of the fiber with high hygroscopicity can raise high thermal stress index of the pilot in high temperature and humidity environment.
\end{abstract}

\section{Introduction}

The Anti-G suit is an important equipment for the fighter pilots, because they have to face high G-Load. Under high G-Load conditions, the blood of the pilot pools in the lower part of the body, thus the brain will be deprived of blood, leading to temporary hypoxia, which causes a black-out and g-LOC (g-induced Loss of Consciousness). Black-out and g-LOC has caused a number of fatal aircraft accidents. In order to minimize the adverse physiological effects of the G-Load, an anti-G suit with bladders is used for the fighter pilot. When the high G-Load takes place, the bladders are fitted and press firmly on the pilot's abdomen and legs, thus restricting the draining of blood away from the brain. Because of impermeable bladders and high thermal resistance, anti-G garments always produce heat stress on wearers even in a moderate environment. The heat stress will reduce pilots' work efficiency and decrease their endurance to the high G-load. To mitigate thermal stress of the pilot in various environments, research on thermal performance of anti-G garment is essential to provide critical information for improving the garment design. Using the model to investigate the thermal performance of the clothing is a very good method. For the pilot/anti-G suit/environment system, Qiu Yifen [1] developed a thermal performance analysis model based on thermal and moisture resistance model of the garment and human bio-heat transfer equations. However, the thermal resistance and moisture resistance are measured under steady-state conditions, which can't fully describe the real heat transfer process within the anti-G garments. Actually, the heat and moisture transfer in the clothing is a dynamic process, in which the fiber hygroscopicity and water content of the fiber has a great effect on the heat and moisture transfer. Li Yi and Li Fengzhi et al[2-4] developed coupled heat and moisture transfer model for the general clothing. In this paper, we develop a coupled heat and moisture transfer model for the anti-G suit with bladders to study the influeces of the fiber hygroscopicity on the pilot heat stress index.

\section{Mathematical Model}

To consider fiber hygroscopicity, the coupled heat and moisture transfer model[3] in the anti-G suit is used. 


$$
\left\{\begin{array}{l}
\frac{\partial\left(\varepsilon_{a} C_{a}\right)}{\partial t}+\varepsilon_{f} \frac{\partial C_{f}}{\partial t}=\frac{\partial}{\partial x}\left(\frac{D_{a} \varepsilon_{a}}{\tau} \frac{\partial C_{a}}{\partial x}\right) \\
c_{v} \frac{\partial T}{\partial t}-\lambda \varepsilon_{f} \frac{\partial C_{f}}{\partial t}=\frac{\partial}{\partial x}\left(k \frac{\partial T}{\partial x}\right) \\
\varepsilon_{a}=1-\varepsilon_{f}
\end{array}\right.
$$

where, $\varepsilon_{a}$ and $\varepsilon_{f}$ are the volumetric fractions of air filling inter-fiber space and fibers, respectively. $C_{a}$ is the water vapor concentration in the air filling inter-fiber space, $C_{f}$ is the mean water vapor concentration in the fibers, which can be obtained by Fick's Law [3]. $D_{a}$ is the diffusion coefficient of water vapor in air, $\tau$ is the effective tortuosity of water vapor through the fabric, $T$ is the fabric temperature, $c_{v}, \lambda$ and $k$ represent the volumetric heat capacity of the fabric, the heat of sorption of water vapor by fibers, and the thermal conductivity of the fabric, respectively. They are functions of water content of the fiber. The water content of the fiber relates to the fiber hygroscopicity.

To consider the bladder effect on the human body temperature, the 25-node Stolwijk's model [5] is improved in this paper. The whole human body is divided into 21 segments, and the abdomen, left front thigh, right front thigh, left front leg and right front leg, which are near the bladders, are separated from the trunk, legs in the Stolwijik's model. Also, each segment is subdivided into 4 layers: core, muscle, fat and skin. Like as the Stolwijk's model, an additional central blood pool representing the large arteries and veins, exchanges heat with all other compartments via the convective heat transfer occurring with the blood flow to each layer. The whole human body is considered as 85-node, so we can call it 85-node model. The heat balance equations for each layer and blood can be written. The equation formulations are similar to the Stolwijik's model, and the evaporation of the skin surface and sweat accumulation are also improved by the Jone's method, more detail information can be found in Ref.[6]. The regulation system for the pilot is same as that of the Stolwijk's model. For describing the body heat stress index, a comprehensive index of heat stress $\mathrm{C}$ was defined by Ref.[1]

$$
C=4.49 \Delta T_{b}+0.1 m_{r s w}
$$

where, $\Delta T_{b}$ is rise of average body temperature, $m_{r s w}$ is the sweat rate $(\mathrm{g} / \mathrm{min}$.).

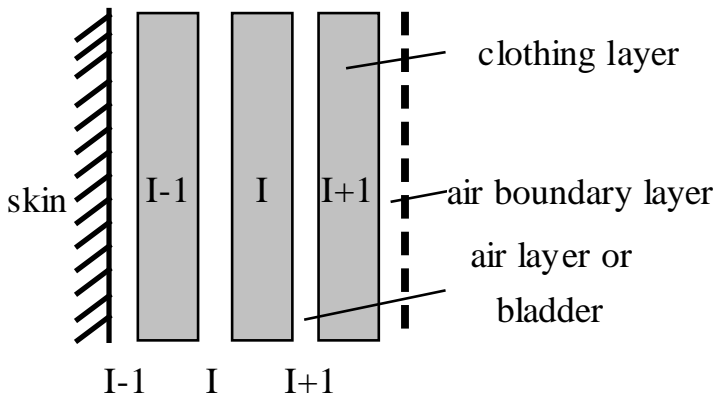

Fig. 1 Pilot skin/anti-G suit/environment-boundary system

To solve the Eq.(1), the initial and boundary conditions must be listed. The initial condition can be given by calculating the temperature and concentration at equilibrium state between clothing and environment. To list the boundary conditions a pilot skin/anti-G suit/environment-boundary system is given as Fig.1. The left boundary conditions on the I-th clothing are given by:

$$
\left\{\begin{array}{l}
-\left.\frac{D_{a} \varepsilon}{\tau} \frac{\partial C_{a}}{\partial x}\right|_{\Gamma I, \text { left }}=H_{m I}\left(C_{a I, \text { left }}-C_{a I-1, \text { right }}\right) \\
-\left.k \frac{\partial T}{\partial x}\right|_{\Gamma I, \text { left }}=H_{t I}\left(T_{I, \text { left }}-T_{I-1, \text { right }}\right)
\end{array} .\right.
$$

and that at right side is 


$$
\left\{\begin{array}{l}
-\left.\frac{D_{a} \varepsilon}{\tau} \frac{\partial C_{a}}{\partial x}\right|_{\Gamma I, \text { right }}=H_{m I+1}\left(C_{a I, \text { right }}-C_{a I+1, l e f t}\right) \\
-\left.k \frac{\partial T}{\partial X}\right|_{\Gamma I, \text { right }}=H_{t I+1}\left(T_{I, \text { right }}-T_{I+1, \text { left }}\right)
\end{array} .\right.
$$

where, $H_{m}$, and $H_{t}$ represent the moisture and heat transfer coefficients of the air or bladder layer. If $\mathrm{I}=1$, then the water vapor flux to clothing equals the evaporation water flux from the skin, and heat flux to clothing equals the sum of the evaporation heat flux, conduction and radiation heat flux from skin. And if $I$ is the outside layer, $C_{a I+1, \text { left }}$ and $T_{I+1, \text { left }}$ are the ambient water vapor concentration and temperature, respectively. If I is outside layer, $H_{t}=h_{r}+h_{c} . h_{r}$ is the radiation heat transfer coefficient. $h_{c}$ is the convective heat transfer coefficient, it is a function of the air velocity $v$, for a seated person, $h_{c}=3.43+5.93 \mathrm{v} \mathrm{W} / \mathrm{m}^{2} \mathrm{~K}$. The moisture transfer coefficient, $H_{m}$, is related to the convection heat transfer, and can be obtained from the Lewis Relation. If a bladder exists between the clothing layer the moisture transfer coefficient is 0 , and heat transfer coefficient equals the conductivity of the bladder.

\section{Model validation}

The numerical technique for human body is explicit differential method, and that for clothing is implicit central differential method. The simulation program is written in FORTRAN. In order to validate the model, we simulated a process of an experiment in a hot climate chamber [1]. The chamber wind velocity is less than $0.1 \mathrm{~m} / \mathrm{s}$ and the vertical and horizontal temperature difference is below $1 \mathrm{C}$. The dry temperature $40 \mathrm{C}$ while the relative humidity is $54 \%$. The volunteers are seated in the climate chamber with anti-G garments without PCM and relevant clothing on. The ant-G garment is made of 2-layer cotton fabric with each $2 \mathrm{~mm}$ thickness and the bladder is made of polyester materials with 3mm thickness. The five bladders are distributed near the abdomen, left front thigh, right front thigh, left front leg and right front leg. The physical parameters can be founded in the Ref.[7] Each volunteer is exposed to the hot chamber environments for minutes. The human body temperature was measured. Human responses are simulated by using the thermoregulation system model under the same experimental conditions. Model predictions are compared to the experimental results, as presented in Fig. 2. Final values of mean skin temperature predicted by modeling and by experiments are consistent with each other. According to the above comparisons the thermal system simulation model is validated to be sufficiently accurate, and could be used for analyzing the thermal performance of anti-G garments.

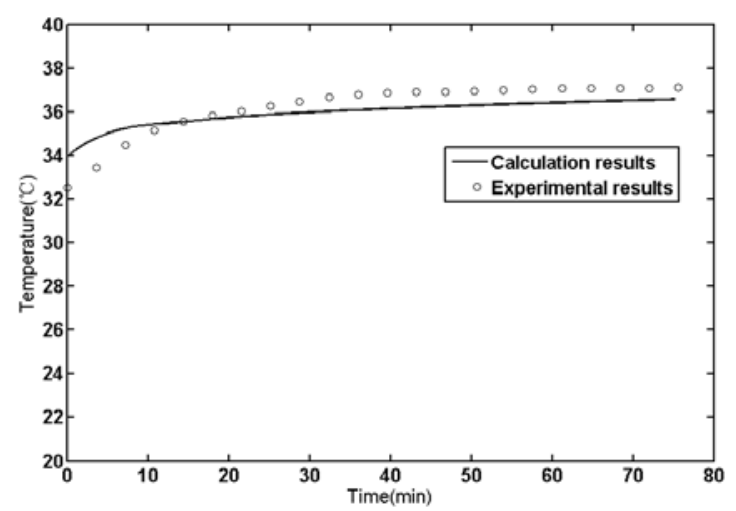

Fig. 2 Comparison of modelling and experimental mean skin temperatures in a $40 \mathrm{C}$ climate chamber

\section{Influences of the fiber hygroscopicity on thermal stress index of the pilot}

After validating the model, we can use it to predict the influences of the fiber hygroscopicity on 
thermal responses of the pilot. The simulation environment is same as the section "Model validation", only the anti-G suit material is changed. We select the wool, cotton and polyester as the basic material of the anti-G suit, respectively. The physical property parameters of the basic materials can be found in the Ref.[7,8]. The differences between the different materials are compared.

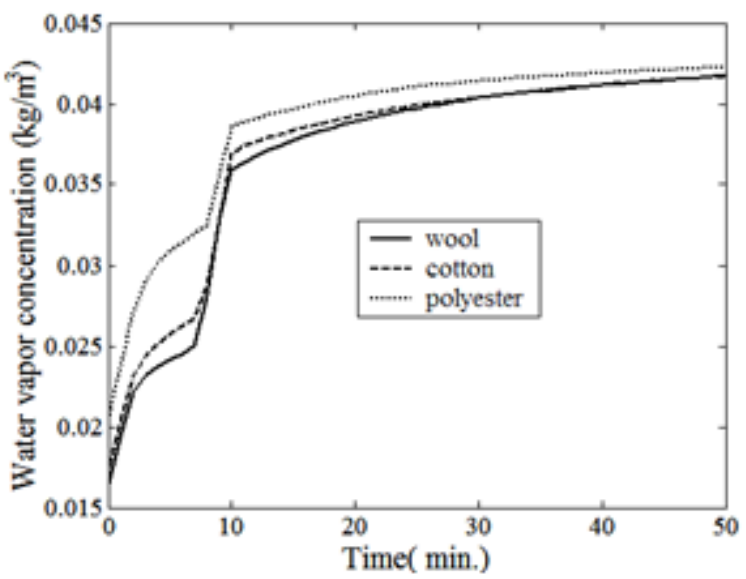

Fig. 3 Water vapor concentration at anti-G suit inner surface near pilot abdomen

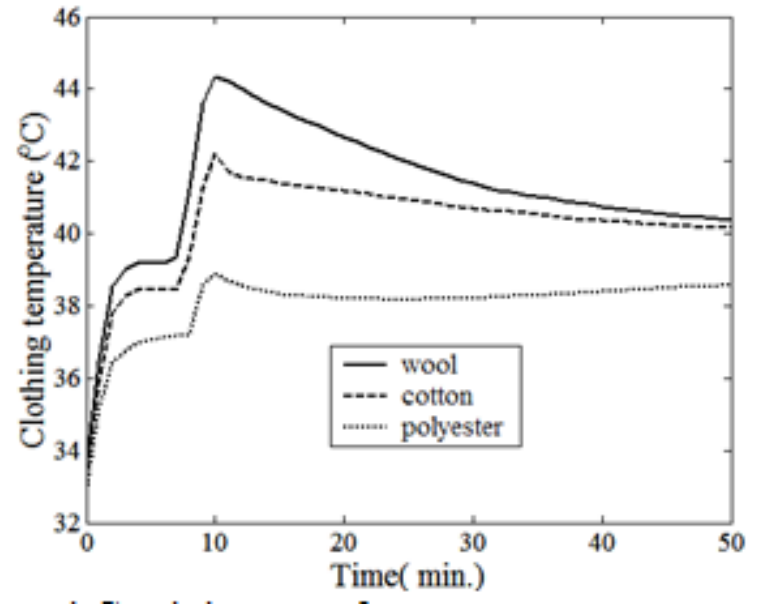

Fig. 4 Temperature at anti-G suit inner surface near pilot abdomen

Fig. 3 shows the water vapor concentration at different anti-G suit inner surface near pilot abdomen. At the beginning the water vapor concentration increases only due to the skin sweat vapor evaporation, because the bladder is impermeable, no water vapor penetrates from outside environment. About 7-th min. the liquid sweat evaporation leads to the concentration increases again. Polyester with the lowest hygroscopicity has the highest water vapor concentration, followed by cotton and wool.

Fig. 4 shows the temperature at anti-G suit inner surface near pilot abdomen. The temperature increases with the environment temperature raise. About 7-th the temperature increases suddenly due to the skin sweat evaporation flux. Also we can see that the woolen anti-G suit has the highest temperature increase in high temperature and humidity environment, followed by cotton and polyester, according to the level of their moisture sorption capacity. The wool fiber has high hygroscopicity and absorbs a large of moisture air, so that the wool fiber releases the more latent heat, and anti-G suit is the highest, followed by the cotton and polyester. 


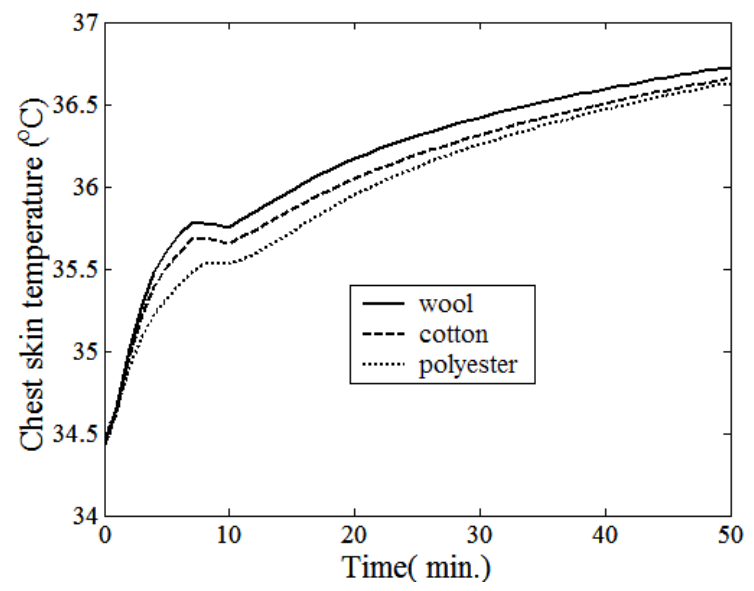

Fig. 5 Chest skin temperature

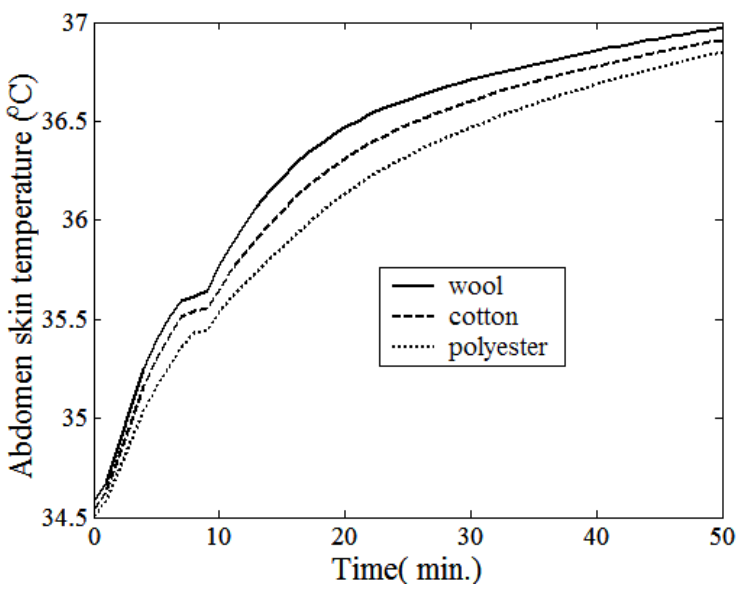

Fig. 6 abdomen skin temperature

Fig.5 and Fig.6 show the chest skin and abdomen skin temperatures, respectively. The skin temperature is the highest when woolen garment is worn, followed by the cotton and polyester.

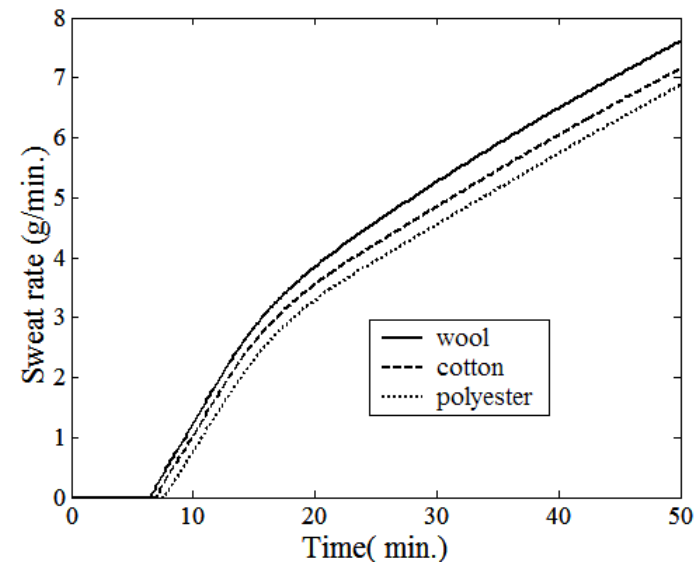

Fig. 7 Sweat rate

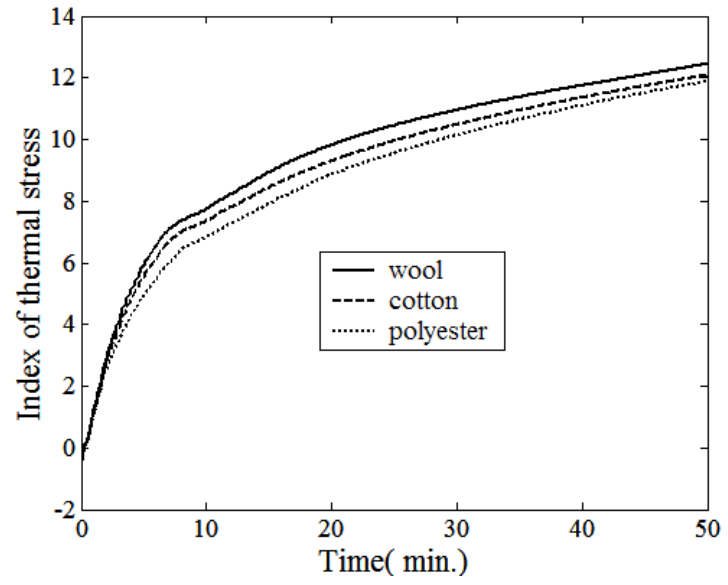

Fig. 8 Index of thermal stress

Fig.7 and Fig.8 show the sweat rate and index of thermal stress of the pilot. All the sweat rate and thermal stress index when wearing different clothing increase during the time period from 0 to the 50-th min. The sweat rate and thermal stress index increase are highest when woolen garment is worn, followed by cotton and polyester.

\section{Summary}

In this paper we develop a heat and moisture transfer model for pilot/anti-G suit/environment system. And the influences of the fiber hygroscopicity on pilot thermal responses are studied by using the model. Conclusion shows that the fiber hygrocopicity has influence on the thermal index of the pilot. Anti-G suit made of wool with high hygroscopicity has high thermal stress index in high temperature and humidity, followed by that made of cotton and polyester.

\section{Acknowledgements}

This work was supported by the Fundamental Research Founds for Central Universities ( NS2013007).

\section{References}

[1] Y. F. Qiu, W. Wu, X. G. Yuan, et al: Chin. J. Mech. Eng. Vol. 25 (2012) , p.47

[2] Y. Li, B. V. Holcombe: Text. Res. J. Vol. 62 (1992). p. 211 
[3] Y. Li, Z. X. Luo: Text. Res. J. Vol. 69 (1999), p.760

[4] F. Z. Li, Y. Li, Y. X. Liu, et al: Numer. Heat Tr. B-Fund. Vol.45 (2004), p. 249

[5] J. A. J. Stolwijk, J. D. Hardy: Pflugers Archiv. Vol. 291 (1966), p.129

[6] B. W. Jones,Y. Ogawa: ASHRAE Trans. Vol. 188(1992) , p. 189

[7] F. Z. Li, Y. Li et al.: Model. Simul. Mater. Sci. Eng. Vol. 13 (2005) , p. 809

[8] Q. Y. Zhu, Y. Li: Int. J. Heat and Mass Tran. Vol.46 (2003), p.5099 